PSS PROCEEDINGS

\title{
Two loop structure of QCD vertices
}

\author{
J.A. Gracey* \\ Theoretical Physics Division, Department of Mathematical Sciences, University of Liverpool, \\ P.O. Box 147, Liverpool, L69 3BX, United Kingdom \\ E-mail: graceyeliv.ac.uk
}

We summarize recent computations of the vertex functions of QCD. These include the full two loop off-shell massless 3-point functions as well as the one loop quartic vertex at the completely symmetric point.

Loops and Legs in Quantum Field Theory - LL 2014,

27 April - 2 May 2014

Weimar, Germany

${ }^{*}$ Speaker. 


\section{Introduction}

Activity in recent years in the study of the perturbative and non-perturbative structure of QCD has concentrated on the vertex functions. At one loop the early work of [1] was useful for Schwinger-Dyson analysis of the $n$-point tower of equations whose solution would in principle provide the full properties of the theory. In practice in order to extract some understanding of the behaviour of the $n$-point functions approximations have to be made and several ansätze for the Lorentz structure of the embedded vertices are used. One is the omission of the quartic vertex. Indeed this assumption invariably appears to be good. However, with the improvement in techniques both analytically and numerically it is now becoming fashionable to question to what extent such assumptions are valid, [2, 3]. Equally lattice studies of vertex functions have improved in recent years to the extent that the infrared behaviour of the vertex functions are forming laboratories for testing ideas for, say, gluon confinement. Therefore to aid both areas of investigation the perturbative structure of the 3-point vertices of QCD have been computed at two loops in various configurations [4, 6, 6, 7, 8, 9, 10, 11, 12, This has primarily been in linear covariant gauges. Though certain nonlinear gauges, [13, 14], have also been analysed. In this article we give a snapshot of recent activity in the perturbative approach to vertex functions studies. In particular we concentrate on the full two loop off-shell 3-point functions of QCD for a general external momentum configuration. Full details are provided in [15]. Such expressions should assist Schwinger-Dyson analyses in exploring the ansätze used therein.

\section{3-point vertices}

The QCD 3-point vertex functions of interest are defined by

$$
\begin{aligned}
\left\langle A_{\mu}^{a}(p) A_{\nu}^{b}(q) A_{\sigma}^{c}(-p-q)\right\rangle & =f^{a b c} \Sigma_{\mu \nu \sigma}^{\mathrm{ggg}}(p, q) \\
\left\langle\psi^{i}(p) \bar{\psi}^{j}(q) A_{\sigma}^{c}(-p-q)\right\rangle & =T_{i j}^{c} \Sigma_{\sigma}^{\mathrm{qgg}}(p, q) \\
\left\langle c^{a}(p) \bar{c}^{b}(q) A_{\sigma}^{c}(-p-q)\right\rangle & =f^{a b c} \Sigma_{\sigma}^{\mathrm{ccg}}(p, q)
\end{aligned}
$$

where $p$ and $q$ are the two independent external momenta and we have factored off the common colour tensor from the Lorentz tensor amplitudes. To use results of basic Feynman integrals which arise in the computation we introduce the two dimensionless variables $x=\frac{p^{2}}{r^{2}}$ and $y=\frac{q^{2}}{r^{2}}$ where $r=-(p+q)$ and the overall mass scale $\mu$ is given by $r^{2}=-\mu^{2}$. We will also use $\mu$ as the scale which ensures that the coupling constant is dimensionless in $d$-dimensions when we dimensionally regularize in $d=4-2 \varepsilon$ dimensions. For contact with [16] we note that the completely symmetric point corresponds to $x=y=1$.

In order to extract the behaviour of the vertices as functions of our variables as well as to have a practical algorithm for a computation we decompose the tensor amplitudes into a basis of Lorentz tensors built from $p, q$, the metric and, in the case of the quark-gluon vertex, the $\gamma$-matrices. For the respective vertices of (2.1) there are respectively 14, 2 and 6 such tensors which are given in [15]. The method to reduce the Lorentz amplitudes to scalar functions is to use a projection, [17]. This means that the constituent Feynman integrals of each vertex function involves at most scalar products of the loop and external momenta. Thus it is in the ideal form for applying the 
Laporta algorithm, [18], which is the current main computational tool for $n$-point functions. For our analysis we have used the REDUZE encoding, [19], and exploited its interface with the symbolic manipulation language FORM, [20, 21]. The Feynman diagrams are generated with the FORTRAN package QGRAF, [22]. In the Laporta approach, which in essence is the algebraic solution of towers of relations between Feynman integrals established by integration by parts, the graphs contributing to a vertex function are reduced to a small set of master integrals. These invariably are determined directly. For the 3-point functions there are five basic integral families for the REDUZE setup. At one loop it is the basic triangle and at two loop there is the non-planar triangle and three ladder graphs. However, in REDUZE the non-bubble masters are in effect one loop triangles. The two loop master integrals correspond to one loop triangle graphs with propagators with non-integer powers. Their $\varepsilon$ expansion is known, [23, 24, 25, 26]. However, due to spurious poles arising from integration by parts the two loop masters are required to $O\left(\varepsilon^{2}\right)$, [15]. Although these are known in harmonic polylogarithm notation, [25], we have extended the $\varepsilon$ expansion of [23, 24, 26] for the full general off-shell case in terms of $\mathrm{Li}_{n}(z)$.

Defining

$$
I(\alpha, \beta, \gamma)=\int_{k} \frac{1}{\left(k^{2}\right)^{\alpha}\left((k-p)^{2}\right)^{\beta}\left((k+q)^{2}\right)^{\gamma}}
$$

then the one loop master is, [23, 24],

$$
I(1,1,1)=-\frac{1}{\mu^{2}}\left[\Phi_{1}(x, y)+\Psi_{1}(x, y) \varepsilon+\left[\frac{\zeta(2)}{2} \Phi_{1}(x, y)+\chi_{1}(x, y)\right] \varepsilon^{2}+O\left(\varepsilon^{3}\right)\right] .
$$

where $\Phi_{1}(x, y)$ involves $\operatorname{Li}_{2}(z)$ and $\Psi_{1}(x, y)$ involves $\operatorname{Li}_{3}(z)$. The unknown function $\chi_{1}(x, y)$ is not required explicitly as it always occurs with a similar term, $\chi_{3}(x, y)$, from the related two loop master. Within all our two loop computations the following combination always emerges

$$
\chi_{3}(x, y)-\chi_{1}(x, y)=\Phi_{2}(x, y)-\frac{1}{2} \ln (x y) \Psi_{1}(x, y)+\frac{1}{4}\left[\ln ^{2}(x)+\ln ^{2}(y)\right] \Phi_{1}(x, y)
$$

where $\Phi_{2}(x, y)$ contains $\mathrm{Li}_{4}(z)$, [23, 24, 26]. The general relation agrees with [26] for a simpler external momentum configuration. For contact with other work, [26, 27], we note that in the notation of both these articles

$$
\chi_{3}(1,1)-\chi_{1}(1,1)=\mathscr{H}_{31}^{(2)}+\mathscr{H}_{43}^{(2)}=\frac{1}{36} \psi^{\prime \prime \prime}\left(\frac{1}{3}\right)-\frac{2 \pi^{4}}{27}
$$

where $\mathscr{H}_{j}^{(n)}$ are the harmonic polylogarithms, [28.

Equipped with this computational algorithm we have determined the full two loop off-shell 3-point vertex functions of QCD in a linear covariant gauge. The full expressions are too long to record here. Though we note that they are not inconsistent with the earlier direct evaluation at the full symmetric point, [17]. Also there are in agreement with another external momentum configuration of [13] which was $x=y=\frac{1}{\omega}, r^{2}=-\omega \mu^{2}$ in the current notation where $\omega$ is an interpolating parameter. More informative is to graphically represent the one and two loop corrections over a range of momenta for $\alpha_{s}=0.125$. This is carried out in Figure 1 for the ghostgluon vertex as an example, where $H_{k}(x, y)=\Sigma_{(k)}^{\operatorname{ccg}}(p, q)$. Clearly at this value of the coupling constant perturbation theory is valid as is evident by the small corrections. The behaviour of the other vertex functions is similar. 

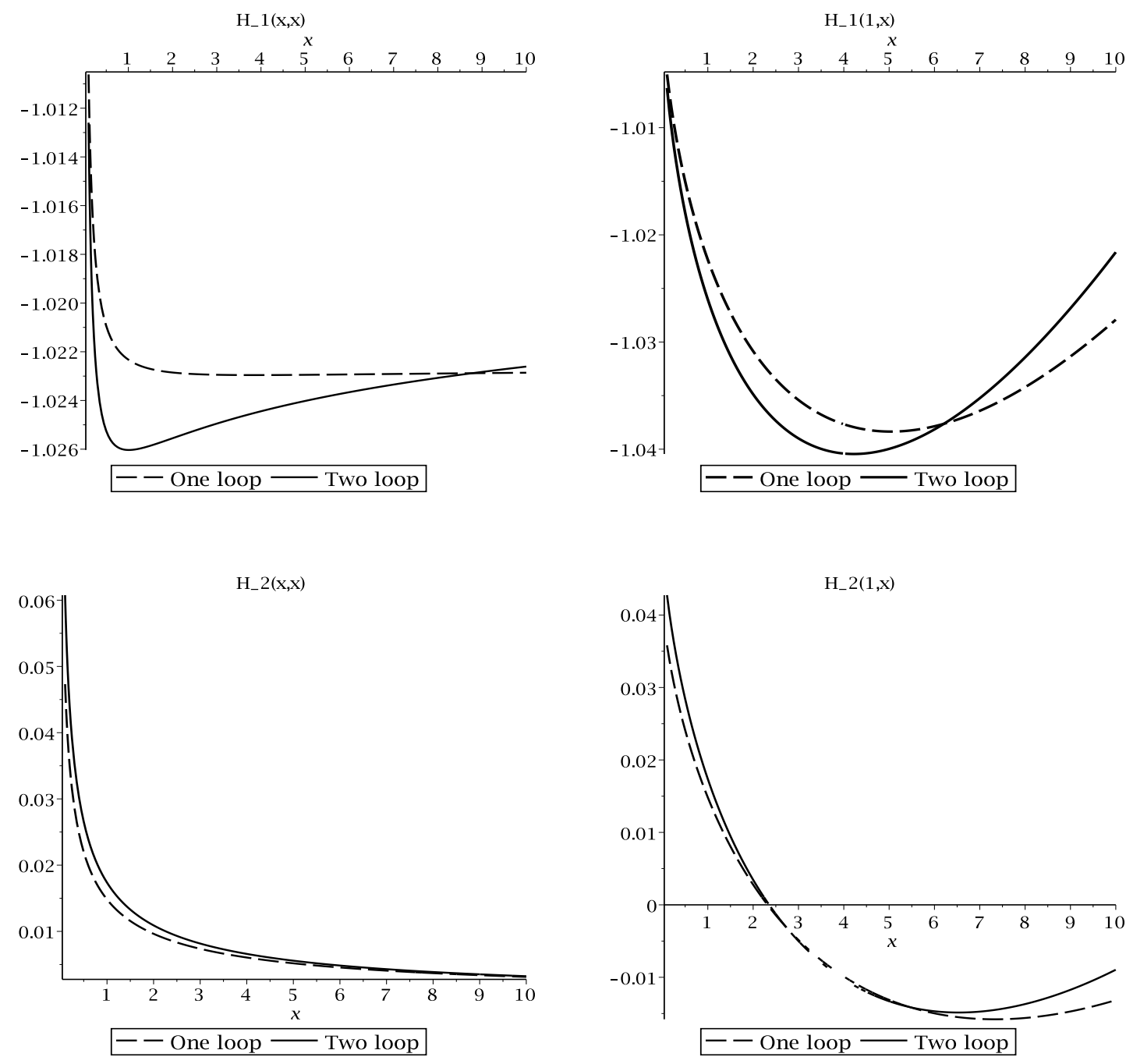

Figure 1: Various sections of the Landau gauge ghost-gluon vertex amplitudes for $\alpha_{s}=0.125$.

Taking the $x \rightarrow 1$ and $y \rightarrow 1$ limits of our general expressions we recover the direct symmetric point renormalization of [17] which extended the previous order computation of [16]. In [16] the momentum subtraction (MOM) renormalization schemes were defined based on each of the trivalent QCD vertices. Also the results of [17] agreed with the earlier numerical estimates of the three loop MOM renormalization group functions of [12]. The latter are constructed as a byproduct of the two loop vertex functions and properties of the renormalization group function. For instance, if one defines the conversion functions from the appropriate renormalization constants, $Z_{k}$, by

$$
C_{g}(a, \alpha)=\frac{Z_{g}^{\mathrm{MOMi}}}{Z_{g}}, C_{\phi}(a, \alpha)=\frac{Z_{\phi}^{\mathrm{MOMi}}}{Z_{\phi}}
$$

where $g$ denotes the coupling constant and $\phi \in\{A, c, \psi\}$ denote the respective fields then for each MOM scheme MOMi

$$
\gamma_{\phi}^{\mathrm{MOMi}}\left(a_{\mathrm{MOMi}}, \alpha_{\mathrm{MOMi}}\right)=\left[\gamma_{\phi}(a)+\beta(a) \frac{\partial}{\partial a} \ln C_{\phi}(a, \alpha)\right.
$$




$$
\left.+\alpha \gamma_{\alpha}(a, \alpha) \frac{\partial}{\partial \alpha} \ln C_{\phi}(a, \alpha)\right]_{\overline{\mathrm{MS}} \rightarrow \mathrm{MOMi}}
$$

where the mapping indicates that $\overline{\mathrm{MS}}$ variables are mapped back to MOMi ones and $a=g^{2} /\left(16 \pi^{2}\right)$ with $g$ the coupling constant. The coupling constants in each scheme are related by

$$
a_{\mathrm{MOMi}}=\frac{a}{\left(C_{g}(a, \alpha)\right)^{2}}
$$

where unlabelled variables are in the $\overline{\mathrm{MS}}$ scheme.

\section{4-point vertex}

With the completion of the two loop off-shell massless 3-point vertices the natural extension is to consider the quartic gluon vertex. Unlike the activity for the former there has been substantially less activity for the 4-point function. One relevant article is [4]. There, for example, the quartic gluon vertex was examined at the fully symmetric point at one loop but in the context of the Weinberg scheme with the focus on that part of the vertex function relevant to the coupling constant renormalization. This has been extended recently in [29] with the motivation to have a quartic MOM scheme in the same class of schemes proposed in [16], as well as information on the full vertex structure. To determine the full one loop symmetric point quartic vertex structure, the same algorithm as that developed for the 3-point functions is used. Although similar we outline the main differences. First, the Green's function is

$$
\left.\left\langle A_{\mu}^{a}(p) A_{v}^{b}(q) A_{\sigma}^{c}(r) A_{\rho}^{d}(-p-q-r)\right\rangle\right|_{\mathrm{symm}}=\left.\Sigma_{\mu \nu \sigma \rho}^{a b c d}(p, q, r)\right|_{\mathrm{symm}}
$$

where symm denotes the completely symmetric quartic point. Specifically the external momenta scalar products are $p^{2}=q^{2}=r^{2}=-\mu^{2}$ and $p q=p r=q r=\frac{1}{3} \mu^{2}$. Next given the extra leg on the vertex function, and thus an additional independent momentum, there are substantially more Lorentz tensors, $\mathscr{P}_{(k) \mu v \sigma \rho}(p, q, r)$, in the basis into which the 4-point function is decomposed. Specifically,

$$
\left.\Sigma_{\mu \nu \sigma \rho}^{a b c d}(p, q, r)\right|_{\mathrm{symm}}=\left.\sum_{k=1}^{138} \mathscr{P}_{(k) \mu v \sigma \rho}(p, q, r) \Sigma_{(k)}^{a b c d}(p, q, r)\right|_{\mathrm{symm}} .
$$

Unlike the 3-point vertices there is no common overall colour group tensor which can be factored off the quartic vertex function. Instead we leave it to be determined for each scalar amplitude from the implementation of the $S U\left(N_{c}\right)$ group generator, $T^{a}$, decomposition

$$
T^{a} T^{b}=\frac{1}{2 N_{c}} \delta^{a b}+\frac{1}{2} d^{a b c} T^{c}+\frac{i}{2} f^{a b c} T^{c}
$$

where $f^{a b c}$ are the structure constants and $d^{a b c}$ is totally symmetric. Using properties of $f^{a b c}$ and $d^{a b c}$ for $S U\left(N_{c}\right)$, [30], we have implemented a FORM module to handle the colour group tensors in such a way that the scalar amplitudes are rewritten in terms of the colour tensor basis $\left\{f^{a b e} f^{c d e}, f^{a c e} f^{b d e}, d_{F}^{a b c d}, d_{A}^{a b c d}\right\}$. The final two tensors are the rank 4 totally symmetric tensors respectively in the fundamental and adjoint representations which were introduced in [31]. Although 
the results of [ [] were expressed in a different colour basis we have chosen this one as it contains the tensor structure of the original quartic gluon Feynman rule as well as having additional tensors reflecting the symmetry of the subtraction point. Moreover, it appears to be the appropriate choice to define the quartic momentum subtraction scheme in the class of schemes of [16]. We will denote this scheme by MOMgggg.

To define the MOMgggg scheme we assume that the full decomposition of the vertex function has been achieved, the underlying Feynman integrals have been evaluated and the scalar amplitudes written in terms of the above colour basis. Then the only renormalization constant to be fixed is that corresponding to the coupling constant. The associated wave function renormalization constants are determined from the one loop 2-point function renormalization in the same manner as [16], in that at the subtraction point there are no $O(a)$ corrections. For the MOMgggg case we follow the same ethos subject to the nature of the Green's function. In other words we define the coupling constant renormalization in such a way that at the symmetric subtraction point there are no $O(a)$ corrections in the colour channels corresponding to the quartic vertex Feynman rule. Once this has been defined it is possible to construct the two loop renormalization group functions by the method outlined earlier. For instance, we find, [29],

$$
\begin{aligned}
\beta^{\operatorname{MOMgggg}}(a, \alpha)=[ & \left.-11 N_{c}+2 N_{f}\right] \frac{a^{2}}{3} \\
+ & {\left[-2592 \ln _{4 / 3} \alpha^{4} N_{c}^{3}+108384 \ln _{4 / 3} \alpha^{3} N_{c}^{3}-3456 \ln _{4 / 3} \alpha^{3} N_{c}^{2} N_{f}\right.} \\
& -408032 \ln _{4 / 3} \alpha^{2} N_{c}^{3}+129536 \ln _{4 / 3} \alpha^{2} N_{c}^{2} N_{f}-56160 \ln _{4 / 3} \alpha N_{c}^{3} \\
& +17280 \ln _{4 / 3} \alpha N_{c}^{2} N_{f}+3159 \Phi_{9 / 16} \alpha^{4} N_{c}^{3}-29943 \Phi_{9 / 16} \alpha^{3} N_{c}^{3} \\
& +4212 \Phi_{9 / 16} \alpha^{3} N_{c}^{2} N_{f}+83889 \Phi_{9 / 16} \alpha^{2} N_{c}^{3}-21672 \Phi_{9 / 16} \alpha^{2} N_{c}^{2} N_{f} \\
& -58305 \Phi_{9 / 16} \alpha N_{c}^{3}+17940 \Phi_{9 / 16} \alpha N_{c}^{2} N_{f}-10800 \Phi_{3 / 4} \alpha^{3} N_{c}^{3} \\
+ & 52800 \Phi_{3 / 4} \alpha^{2} N_{c}^{3}-14400 \Phi_{3 / 4} \alpha^{2} N_{c}^{2} N_{f}-26000 \Phi_{3 / 4} \alpha N_{c}^{3} \\
& +8000 \Phi_{3 / 4} \alpha N_{c}^{2} N_{f}-10080 \alpha^{4} N_{c}^{3}+94560 \alpha^{3} N_{c}^{3}-13440 \alpha^{3} N_{c}^{2} N_{f} \\
& -261280 \alpha^{2} N_{c}^{3}+67840 \alpha^{2} N_{c}^{2} N_{f}+176800 \alpha N_{c}^{3}-54400 \alpha N_{c}^{2} N_{f} \\
& \left.-870400 N_{c}^{3}+332800 N_{c}^{2} N_{f}-76800 N_{f}\right] \frac{a^{3}}{76800 N_{c}}+O\left(a^{4}\right)
\end{aligned}
$$

where

$$
\ln _{4 / 3} \equiv \ln \left(\frac{4}{3}\right), \Phi_{3 / 4} \equiv \Phi_{1}\left(\frac{3}{4}, \frac{3}{4}\right), \Phi_{9 / 16} \equiv \Phi_{1}\left(\frac{9}{16}, \frac{9}{16}\right) .
$$

The final two objects arise from two basic master integrals which emerge from the REDUZE database. They derive from a triangle graph where one leg corresponds to the Mandelstam variable $s$ and the other is the pure scalar symmetric box given in [32]. As far as we can check we find agreement with results of [ 4 ] aside from a few typographical errors. The complete analysis is given in [29].

\section{Discussion}

We conclude with several brief remarks. First, the full two loop off-shell 3-point vertex func- 
tions of massless QCD are now available, [15]. One motivation for that analysis, aside from the connection with Schwinger-Dyson equations, is to assist with lattice measurements and matching onto the high energy behaviour. Although the focus here has been on linear covariant gauges it has been extended to various nonlinear gauges, [13, 14, as these are also of interest in understanding the low energy properties of Yang-Mills theories. Equally the symbolic manipulation code can be applied to other problems such as the renormalization of operators, [13], underlying proton structure functions again motivated by lattice considerations. Finally, the first step towards to completing the same analysis for the quartic gluon vertex has been taken here. Although a two loop analysis even at the fully symmetric point would be a massive undertaking it appears to be viable given the technical advances in recent years such as the Laporta algorithm.

\section{References}

[1] J.S. Ball \& T.W. Chiu, Analytic properties of the vertex function in gauge theories 1, Phys. Rev. D22 (1980) 2542; Analytic properties of the vertex function in gauge theories 2, Phys. Rev. D22 (1980) 2550.

[2] A.L. Blum, M.Q. Huber, M. Mitter \& L. von Smekal, Gluonic three-point correlations in pure Landau gauge QCD, Phys. Rev. D89 (2014) 061703 [arXiv: 1401.0713 ].

[3] G. Eichmann, R. Williams, R. Alkofer \& M. Vujinovic, The three-gluon vertex in Landau gauge, Phys. Rev. D89 (2014) 105014 [arXiv: 1402 . 1365].

[4] P. Pascual \& R. Tarrach, Slavnov-Taylor identities in Weinberg's renormalization scheme, Nucl. Phys. B174 (1980) 123; Nucl. Phys. B181 (1981) 546(E).

[5] A.I. Davydychev, P. Osland \& O.V. Tarasov, Three gluon vertex in arbitrary gauge and dimension, Phys. Rev. D54 (1996) 4087 [hep-ph / 960534 ]; Phys. Rev. D59 (1999) 109901(E).

[6] A.I. Davydychev, P. Osland \& L. Saks, Quark mass dependence of the one loop three gluon vertex in arbitrary dimension, JHEP 0108 (2001) 050 [hep-ph / 0105072 ].

[7] M. Binger \& S.J. Brodsky, The form-factors of the gauge-invariant three-gluon vertex, Phys. Rev. D74 (2006) 054016 [hep-ph / 0602199 ].

[8] A.I. Davydychev, P. Osland \& O.V. Tarasov, Two loop three gluon vertex in zero momentum limit, Phys. Rev. D58 (1998) 036007 [hep-ph / 9801380 ].

[9] A.I. Davydychev \& P. Osland, On-shell two loop three gluon vertex, Phys. Rev. D59 (1998) 014006 [hep-ph/9806522].

[10] A.I. Davydychev, P. Osland \& L. Saks, Quark gluon vertex in arbitrary gauge and dimension, Phys. Rev. D63 (2000) 014022 [hep-ph/ 0008171$].$

[11] K.G. Chetyrkin \& A. Rétey, Three loop three linear vertices and four loop similar to $\widetilde{M O M} \beta$ functions in massless QCD, hep-ph/0007088 [hep-ph/0007088].

[12] K.G. Chetyrkin \& T. Seidensticker, Two loop QCD vertices and three loop MOM beta functions, Phys. Lett. B495 (2000) 74 [hep-ph/ 0008094 ].

[13] J.M. Bell \& J.A. Gracey, paper in preparation (2014).

[14] J.M. Bell \& J.A. Gracey, Momentum subtraction scheme renormalization group functions in the maximal Abelian gauge, Phys. Rev. D88 (2013) 085027 [arXiv: 1310 . 0243]. 
[15] J.A. Gracey, Off-shell two loop QCD vertices, arXiv:1406.0649 [arXiv: 1406.0649 ].

[16] W. Celmaster \& R.J. Gonsalves, The renormalization prescription dependence of the QCD coupling constant, Phys. Rev. D20 (1979) 1420.

[17] J.A. Gracey, Two loop QCD vertices at the symmetric point, Phys. Rev. D84 (2011) 085011 [arXiv:1108.4806].

[18] S. Laporta, High precision calculation of multiloop Feynman integrals by difference equations, Int. J. Mod. Phys. A15 (2000) 5087 [hep-th/ 0207004 ].

[19] C. Studerus, Reduze - Feynman integral reduction in C++, Comput. Phys. Commun. 181 (2010) 1293 [arXiv:0912.2546].

[20] J.A.M. Vermaseren, New features of FORM, math-ph/0010025 [math-ph/0010025].

[21] M. Tentyukov \& J.A.M. Vermaseren, The multithreaded version of FORM, Comput. Phys. Commun. 181 (2010) 1419 [hep-ph/0702279].

[22] P. Nogueira, Automatic Feynman graph generation, J. Comput. Phys. 105 (1993) 279.

[23] N.I. Ussyukina \& A.I. Davydychev, Some exact results for two loop diagrams with three and four external lines, Phys. Atom. Nucl. 56 (1993) 1553 [hep-ph/9307327].

[24] N.I. Ussyukina \& A.I. Davydychev, New results for two loop off-shell three point diagrams, Phys. Lett. B332 (1994) 159 [hep-ph/9402223].

[25] T.G. Birthwright, E.W.N. Glover \& P. Marquard, Master integrals for massless two-loop vertex diagrams with three offshell legs, JHEP 0409 (2004) 042 [hep-ph / 0407343 ].

[26] M. Gorbahn \& S. Jäger, Precise $\overline{M S}$ light-quark masses from lattice QCD in the RI/SMOM scheme, Phys. Rev. D82 (2010) 114001 [arXiv: 1004 . 3997].

[27] L.G. Almeida \& C. Sturm, Two-loop matching factors for light quark masses and three-loop mass anomalous dimensions in the RI/SMOM schemes, Phys. Rev. D82 (2010) 054017 [arXiv: 1004.4613$]$.

[28] E. Remiddi \& J.A.M. Vermaseren, Harmonic polylogarithms, Int. J. Mod. Phys. A15 (2000) 725 [hep-ph/9905237].

[29] J.A. Gracey, Symmetric point quartic gluon vertex and momentum subtraction, arXiv:1406.1618 [arXiv: 1406.1618$]$.

[30] A.J. Macfarlane, A. Sudbery \& P.H. Weisz, On Gell-Mann's $\lambda$-matrices, $d$ - and f-tensors, octets, and parametrizations of $S U(3)$, Commun. Math. Phys. 11 (1968) 77.

[31] T. van Ritbergen, A.N. Schellekens \& J.A.M. Vermaseren, Group theory factors for Feynman diagrams, Int. J. Mod. Phys. A14 (1999) 41 [hep-ph / 9802376$].$

[32] N.I. Ussyukina \& A.I. Davydychev, Exact results for three and four point ladder diagrams with an arbitrary number of rungs, Phys. Lett. B305 (1993) 136. 